The Astrophysical JouRNAL, 547:231-239, 2001 January 20

(C) 2001. The American Astronomical Society. All rights reserved. Printed in U.S.A.

\title{
ULTRA-LITHIUM-DEFICIENT HALO STARS AND BLUE STRAGGLERS: A COMMON ORIGIN? ${ }^{1}$
}

\author{
SEAN G. RYAN \\ Department of Physics and Astronomy, Open University, Walton Hall, Milton Keynes MK7 6AA, England, UK; s.g.ryan@open.ac.uk \\ TIMOTHY C. BEERS \\ Department of Physics and Astronomy, Michigan State University, East Lansing, MI 48824; beers@pa.msu.edu \\ TOSHITAKa KAJINO \\ National Astronomical Observatory of Japan, Osawa 2-21-1, Mitaka, Tokyo 181-8588, Japan; and Department of Astronomy, \\ University of Tokyo, 7-3-1 Hongo, Bunkyo-ku, Tokyo 113-0015, Japan; kajino@ferio.mtk.nao.ac.jp \\ AND \\ KATARINA Rosolankova \\ Department of Physics and Astronomy, Open University, Walton Hall, Milton Keynes MK7 6AA, England, UK; \\ katarina.rosolankova@,st-hildas.oxford.ac.uk \\ Received 2000 April 7 ; accepted 2000 September 21
}

\begin{abstract}
We present data for four ultra-Li-deficient, warm, halo stars. The Li deficiency of two of these is a new discovery. Three of the four stars have effective temperatures $T_{\text {eff }} \sim 6300 \mathrm{~K}$, in contrast to previously known Li-deficient halo stars, which spanned the temperature range of the Spite plateau. In this paper we propose that these and previously known ultra-Li-deficient halo stars may have had their surface lithium abundances reduced by the same mechanism as produces halo field blue stragglers. Even though these stars have yet to reveal themselves as blue stragglers, they might be regarded as "bluestragglers-to-be." In our proposed scenario, the surface abundance of Li in these stars could be destroyed (1) during the normal pre-main-sequence single-star evolution of their low-mass precursors, (2) during the post-main-sequence evolution of an evolved mass donor, and/or (3) via mixing during a masstransfer event or stellar merger. The warmest Li-deficient stars at the turnoff would be regarded as emerging "canonical" blue stragglers, whereas cooler ones represent sub-turnoff-mass blue-stragglers-tobe. The latter are presently hidden on the main sequence, Li depletion being possibly the clearest signature of their past history and future significance. Eventually, the main-sequence turnoff will reach down to their mass, exposing those Li-depleted stars as canonical blue stragglers when normal stars of that mass evolve away. Arguing against this unified view is the observation that the three Li-depleted stars at $T_{\text {eff }} \simeq 6300 \mathrm{~K}$ are all binaries, whereas very few of the cooler systems show evidence for binarity; it is thus possible that two separate mechanisms are responsible for the production of Li-deficient mainsequence halo stars.
\end{abstract}

Subject headings: binaries: spectroscopic - blue stragglers - early universe - Galaxy: halo stars: abundances - stars: Population II

\section{INTRODUCTION}

${ }^{7} \mathrm{Li}$ is destroyed in stellar interiors where temperatures exceed $2.5 \times 10^{6} \mathrm{~K}$, and Li-depleted material can in principle reach the stellar surfaces, where it can be observed. Thus, if one is to infer prestellar ${ }^{7} \mathrm{Li}$ abundances from current-epoch observations, it is important to understand the stellar processing of this species. It has widely, though not universally, been supposed that warm $\left(T_{\text {eff }}>5700 \mathrm{~K}\right)$, metal-poor $([\mathrm{Fe} / \mathrm{H}]<-1)$ stars retain their prestellar abundances (Spite \& Spite 1982; Bonifacio \& Molaro 1997; but see also Deliyannis 1995; Ryan et al. 1996). Although claims had been made of an intrinsic spread in the Li abundances by $0.04-0.1$ dex (Deliyannis, Pinsonneault, \& Duncan 1993; Thorburn 1994), Ryan, Norris, \& Beers (1999) attributed these to an embedded $A(\mathrm{Li})$ versus $[\mathrm{Fe} / \mathrm{H}]$ dependence and underestimated errors, respectively. Ryan

\footnotetext{
${ }^{1}$ Based on observations obtained with the University College London échelle spectrograph (UCLES) on the Anglo-Australian Telescope (AAT) and the Utrecht échelle spectrograph (UES) on the William Herschel Telescope (WHT).
}

et al. (1999) set tight limits on the intrinsic spread of ${ }^{7} \mathrm{Li}$ in metal-poor field stars as essentially zero, stated conservatively as $\sigma_{\text {int }}<0.02$ dex. However, the subset of ultra-Lideficient stars identified by Spite, Maillard, \& Spite (1984), Hobbs \& Mathieu (1991), Hobbs, Welty, \& Thorburn (1991), Thorburn (1992), and Spite et al. (1993) stands out as a particularly exceptional counterexample to the general result. These stars have only upper limits on their ${ }^{7} \mathrm{Li}$ abundances, typically 0.5 dex or more below otherwise similar stars of the same $T_{\text {eff }}$ and metallicity. Detailed studies of other elements in these objects have revealed some chemical anomalies but none common to all or which might explain why their $\mathrm{Li}$ abundances differ so clearly from those of otherwise similar stars (Norris et al. 1997c; Ryan, Norris, \& Beers 1998).

In contrast to the situation for Population II stars, a wider range of $\mathrm{Li}$ behaviors is seen in Population I. In addition to a stronger increase with metallicity, thought to be due to the greater production of $\mathrm{Li}$ in later phases of Galactic chemical evolution (Ryan et al. 2001), there is substantial evidence of $\mathrm{Li}$ depletion in certain temperature ranges. Open cluster observations, for example, show steep 
dependences on temperature for $T_{\text {eff }} \lesssim 6000 \mathrm{~K}$ (e.g., Hobbs \& Pilachowski 1988) and in the region of the F-star Li gap (6400 K $<T_{\text {eff }}<7000 \mathrm{~K}$; Boesgaard \& Tripicco 1986). More problematic, for the young cluster $\alpha$ Per (age $50 \mathrm{Myr}$ ) and the Pleiades (age $100 \mathrm{Myr}$ ), is the presence of a large apparent Li spread even at a given mass. Various explanations have been proposed involving mixing in addition to that due to convection. Extra mixing processes include rotationally induced mixing (e.g., Chaboyer, Demarque, \& Pinsonneault 1995), structural changes associated with rapid rotation (Martín \& Claret 1996), and different degrees of suppression of mixing by dynamo-induced magnetic fields (Ventura et al. 1998). Gravity waves have been proposed as yet another different mixing mechanism (Schatzman 1993; Montalbán \& Schatzman 1996). Consensus has not yet emerged concerning the range of possible mechanisms or the relative importance of each. Jeffries (1999) even questions the reality of a Li abundance spread in low-mass Pleiades stars, due to a similar spread being seen in the K I resonance line. Amongst older open clusters, the spread at a given effective temperature is generally much less, though M67 (Jones, Fischer, \& Soderblom 1999) is an exception. A class of stars with higher lithium abundances than otherwise similar stars is short-period tidally locked binaries (Deliyannis et al. 1994; Ryan \& Deliyannis 1995), which give credence to the view that physics related to stellar rotation can and does influence the evolution of $\mathrm{Li}$ in approximately solar-mass stars.

The fraction of warm, metal-poor stars that fall in the ultra-Li-deficient category has previously been estimated at approximately 5\% (Thorburn 1994). However, recent measurements of $\mathrm{Li}$ in a sample of 18 warm $\left(T_{\text {eff }} \gtrsim 6000 \mathrm{~K}\right)$, metal-poor $(-2 \lesssim[\mathrm{Fe} / \mathrm{H}] \lesssim-1)$ stars yielded four ultraLi-deficient objects, i.e., more than $20 \%$ of the sample (Ryan et al. 2001). The Poisson probability of a $5 \%$ population yielding four or more objects in a sample of this size is just 0.013. Clearly, the selection criteria for this sample have opened up a regime rich in ultra-Li-poor stars. We now examine those criteria and discuss the implications for the origin of such systems and for our understanding of $\mathrm{Li}$-poor and $\mathrm{Li}$-normal stars.

We note some similarities between Li-deficient halo stars and blue stragglers. Although these two groups have traditionally been separated due to the different circumstances of their discovery, we question whether there is a reliable astrophysical basis for this separation. One must ask whether the process(es) that gives rise to blue stragglers is capable only of producing stars whose mass is greater than that of the main-sequence turnoff of a $\sim 13 \mathrm{Gyr}$ old population. If, as we think is reasonable, the answer is "no," then one may ask what the subturnoff-mass products of this process(es) would be. Our proposal is that they would be Li-deficient, but otherwise difficult to distinguish from the general population and, in this regard, very similar to the ultra-Lideficient halo stars.

\section{OBSERVATIONS OF THE ULTRA-Li-POOR HALO STARS}

The ultra-Li-poor halo stars that we consider were identified serendipitously in a study of predominantly high proper motion halo stars having $T_{\text {eff }} \gtrsim 6000 \mathrm{~K}$ and $-2 \lesssim[\mathrm{Fe} / \mathrm{H}] \lesssim-1$, and are listed in part A of Table 1 . Details of the sample selection and abundance analysis are given by Ryan et al. (2001); the key points are that high resolving power $(\lambda / \Delta \lambda \simeq 50000)$ échelle spectra were obtained, equivalent widths were measured, and abundances were computed using a model atmosphere spectrum-synthesis approach. Two of the Li-poor stars were subsequently found to have previous $\mathrm{Li}$ measurements: Wolf 550 was identified as G66-30 and G202-65 had been observed by Hobbs \& Mathieu (1991) in a study targeted at blue stragglers. The new spectra of the four stars, plus one with normal $\mathrm{Li}$ for comparison, are shown in Figure 1. The comparison star, CD $-31^{\circ} 305$, has $T_{\text {eff }}=$ $5970 \mathrm{~K},[\mathrm{Fe} / \mathrm{H}]=-1.0$, and $A(\mathrm{Li})=2.24$ (Ryan et al. 2001). For convenience, previously known Li-depleted halo stars are listed in part B of Table 1. The full sample of Ryan et al. (2001) is plotted in Figure 2, along with additional stars from the literature.

It is immediately apparent that three of the four ultra-Lideficient stars are amongst the hottest in our sample, though not the hottest in the figure. It seems likely that high temperature is one biasing characteristic of these objects. The stars with $T_{\text {eff }}>6300 \mathrm{~K}$ and normal Li abundances are listed in part $\mathrm{C}$ of Table 1 . These have had comparatively high values of dereddening applied, and it is possible that they are in reality cooler than Table 1 shows. An indication that high temperature is not the only biasing characteristic of ultra-Li-poor stars is that the Ryan et al. (1999) study of

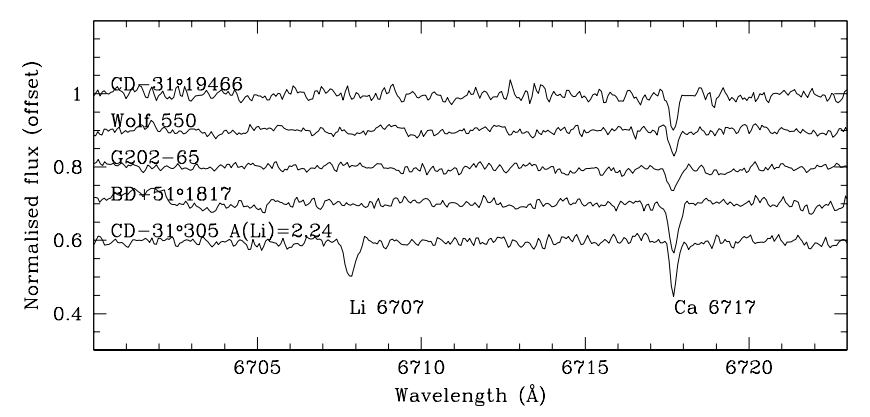

FIG. 1.- Spectra in the region of the Li 6707 doublet, in order of increasing $[\mathrm{Fe} / \mathrm{H}]$. A fifth star, $\mathrm{CD}-31^{\circ} 305$, with $\mathrm{Li}$ abundance close to the Spite plateau, is shown for comparison.

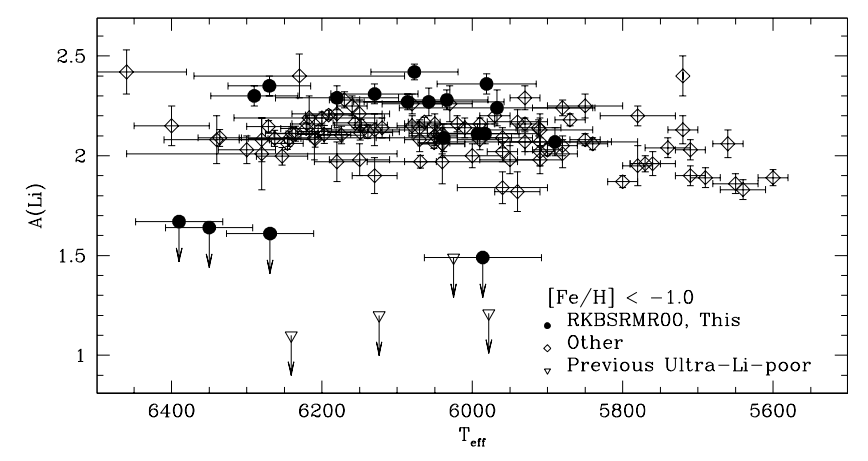

FIG. 2.-Variation of $A(\mathrm{Li})$ with $T_{\text {eff }}$ for halo dwarfs with $[\mathrm{Fe} / \mathrm{H}]<-1.0$. Three of the four ultra-Li-depleted stars are amongst the hottest in the sample, even though stars down to $T_{\text {eff }}=5900 \mathrm{~K}$ were included. Filled circles, Ryan et al. (2001) and this study; open triangles, previous Li-deficient observations (see Table 1); open diamonds, data from Rebolo, Molaro, \& Beckman (1988), the compilation by Ryan et al. (1996), Ryan et al. (1999), Norris, Beers, \& Ryan (2000), and Spite et al. (2000). 


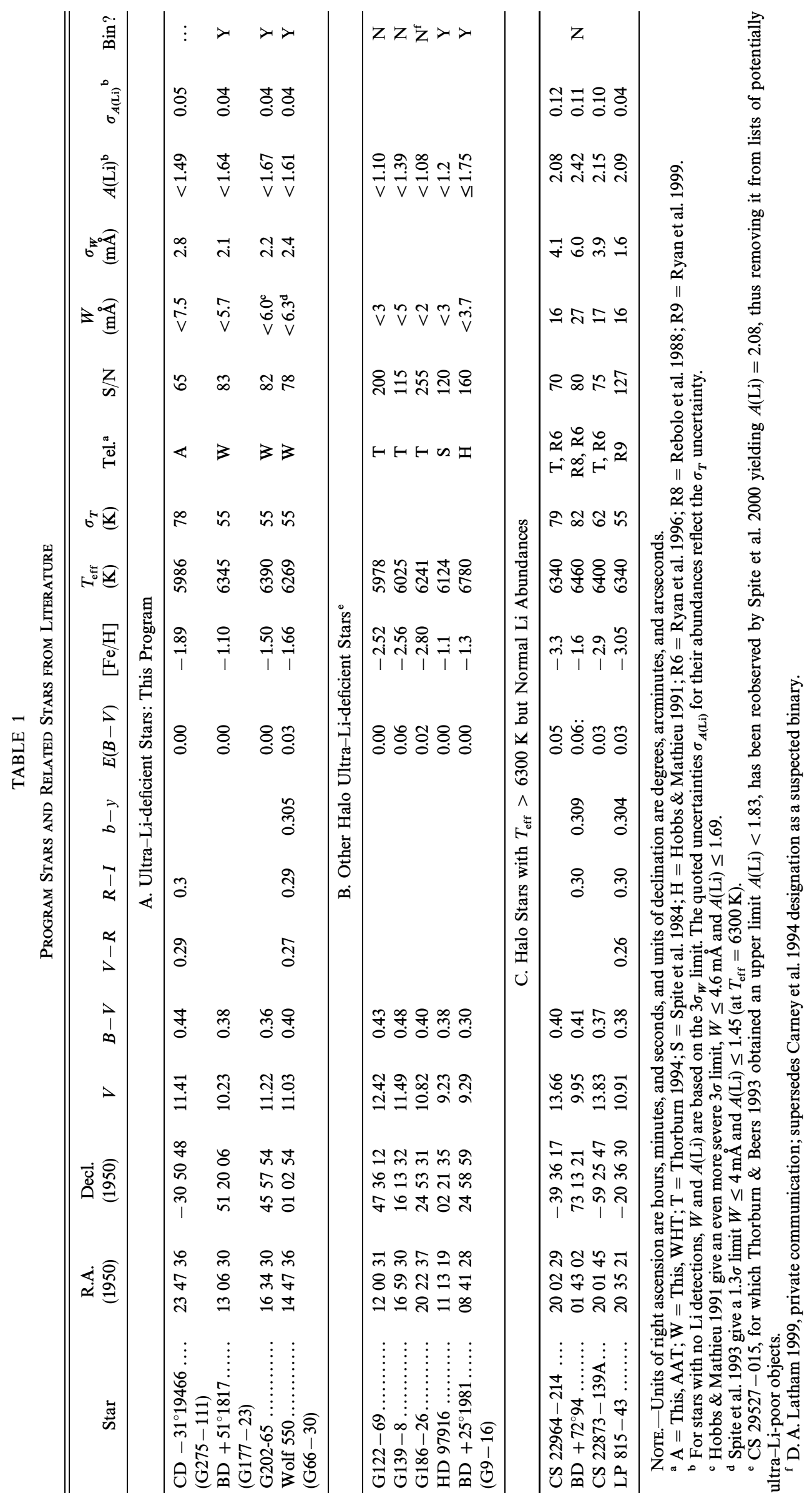


23 very metal-poor $(-3.5 \lesssim[\mathrm{Fe} / \mathrm{H}] \lesssim-2.5)$ stars in the same temperature range included only one ultra-Lideficient star, G186-26. This rate, 1 in 23 , is consistent with previous estimates for Population II stars as a whole. However, very few relatively metal-rich $(-2 \lesssim[\mathrm{Fe} / \mathrm{H}] \lesssim$ -1 ) halo stars in this temperature range had been studied previously, so earlier works may have been biased against discovering ultra-Li-poor objects. It appears, then, that the fraction of ultra-Li-deficient stars is higher as metallicity increases. This may explain why our study, which targeted stars in the higher metallicity range and with $T_{\text {eff }}>6000 \mathrm{~K}$, was so successful at yielding ultra-Li-deficient stars. Figure 3 shows the distribution of objects in the $T_{\text {eff }}-[\mathrm{Fe} / \mathrm{H}]$ plane. Whereas previously no region of parameter space stood out as "preferred" by Li-deficient stars, the objects are now more conspicuous as a result of their high temperatures and relatively high metallicities.

Also shown in Figure 3 are the $T_{\text {eff }}$ of the main-sequence turnoff as a function of metallicity for 14 and 18 Gyr isochrones. The isochrones shown are the oxygen-enhanced curves of Bergbusch \& VandenBerg (1992; solid curves; $Y=0.235$ ) and, for comparison, the Revised Yale isochrones of Green, Demarque, \& King (1987; dotted curves; $Y=0.24$ ). Clearly, there is disagreement of $\simeq 4 \mathrm{Gyr}$ between the two sets as to the ages that would be assigned to these stars, and there are uncertainties in the color- $T_{\text {eff }}$ transformations that have been applied to the observed data. However, these difficulties are not the issue here. Rather, we use the isochrones to indicate the shape of the turnoff locus in the $T_{\text {eff }}-[\mathrm{Fe} / \mathrm{H}]$ plane, and on that point the four isochrones are in overall agreement. They emphasize that even though HD 97916 is cooler than five other Lidepleted stars in the study, it is nevertheless close to the turnoff. That is, a star with $T_{\text {eff }}=6100 \mathrm{~K}$ would appear below the turnoff if $[\mathrm{Fe} / \mathrm{H}]=-3$ but will be close to the

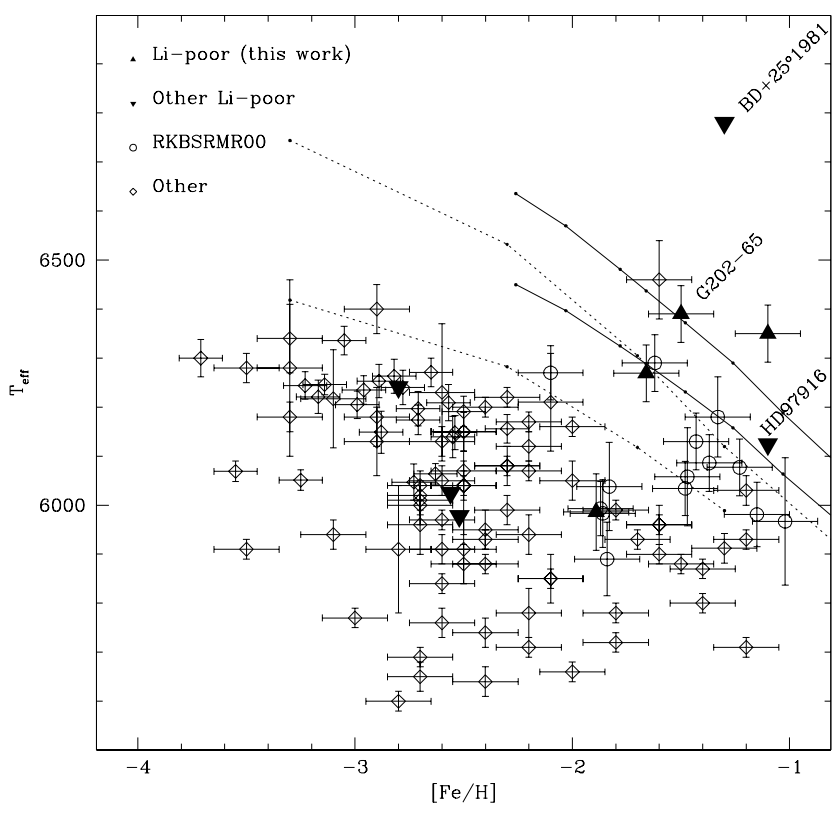

FIG. 3.-Location of halo stars with known $A(\mathrm{Li})$ in the $T_{\text {eff }}-[\mathrm{Fe} / \mathrm{H}]$ plane. Filled triangles, ultra-Li-deficient stars; open circles, Ryan et al. (2001); open diamonds, as in Fig. 2. The locus of turnoff stars of different metallicity are shown for $14 \mathrm{Gyr}$ and $18 \mathrm{Gyr}$ isochrones from Bergbusch \& VandenBerg (1992; solid curve) and Green et al. (1987; dotted curve). turnoff if $[\mathrm{Fe} / \mathrm{H}]=-1$. Even excluding the definite blue straggler $\mathrm{BD}+25^{\circ} 1981$, there are four Li-depleted stars amongst the eight whose symbols lie above or touch the 14 Gyr Revised Yale isochrone. Clearly, all of these are very close to the turnoff once their metallicities are taken into account. ${ }^{2}$ Besides these Li-depleted stars close to the turnoff, four are $100-200 \mathrm{~K}$ cooler than the turnoff. We discuss later in this paper whether these two groupings might have different origins.

\section{TRADITIONAL BLUE STRAGGLERS}

Blue stragglers are recognized observationally as stars that are considerably bluer than the main-sequence turnoff of the population to which they belong, but have a luminosity consistent with main-sequence membership. Such objects were originally identified in globular clusters (e.g., M3; Sandage 1953) but are also known in the field (e.g., Carney \& Peterson 1981) and in Population I as well as Population II (e.g., Leonard 1989; Stryker 1993). Their origin is not known with certainty, and it is possible that more than one mechanism is responsible for their presence. A range of explanations was examined by Leonard (1989), but the discovery of Li destruction in blue stragglers in the halo field and the open cluster M67 led Hobbs \& Mathieu (1991) and Pritchet \& Glaspey (1991) to conclude that "virtually all mechanisms for the production of blue stragglers other than mixing, binary mass transfer, or binary coalescence appear to be ruled out ...." As Hobbs \& Mathieu emphasized, internal mixing alone is also ruled out; mixing out to the surface is required.

Recent advances in high-resolution imaging have verified that the blue straggler fractions in at least some globular clusters are higher in their cores, strongly supporting the view that some blue stragglers are formed through stellar collisions, probably involving the coalescence of binary stars formed and/or hardened through exchanges, in these dense stellar environments (e.g., Ferraro et al. 1999). However, it is neither established nor required that a single mechanism will explain all blue stragglers, and it is unclear how the field examples and those in the tenuous dwarf galaxy Ursa Minor (S. Feltzing 2000, private communication) relate to those in the dense cores of globular clusters. Probably even the halo field and dwarf spheroidal stars formed in clusters of some description (since the formation of stars in isolation is unlikely), but one should not be too quick to link the properties of surviving globular clusters to diffuse populations. This view is supported by Preston \& Sneden's (2000) conclusion that more than half $(62 \%-100 \%)$ of their field blue metal-poor binaries are blue stragglers formed by mass transfer rather than mergers, due to the long orbital periods and low eccentricities of the field systems they observed. Their conclusion is entirely consistent with the views of Ferraro, Fusi Pecci, \& Bellazini (1995), who ascribed blue straggler formation to interactions between systems in high-density environments, but within systems (primordial binaries) in lower density clusters. In contrast to but not contradicting Preston \&

\footnotetext{
${ }^{2}$ We resist the temptation to speak of a single locus for the turnoff because of the possibility that an age spread exists at a given metallicity. That issue has not yet been settled for the globular clusters (see Piotto 2000 and Chaboyer 2000), despite those systems being better constrained. For the same reason, and because of random errors in the effective temperature estimates, we refrain from debating whether a particular star lying close to the turnoff is definitely above or below the turnoff.
} 
Sneden's result for field systems, Mateo et al. (1990) argue that all of the blue stragglers in the globular cluster NGC 5466 are the result of close binary mergers.

The mechanism for Li destruction in field blue stragglers is not known. It is unclear what degree of mixing will occur as a result of coalescence. Early work by Webbink (1976) suggested substantial mixing would occur, whereas more recent simulations of head-on collisions by Sills et al. (1997) and grazing collisions and binary mergers by Sandquist, Bolte, \& Hernquist (1997) have suggested otherwise. Sills, Bailyn, \& Demarque (1995) argue, however, that to account for the blue stragglers observed in NGC 6397, mixing is nevertheless required (unless the collision products have more than twice the turnoff mass) and may occur after the initial coalescence. This is perhaps consistent with the result of Lombardi, Rasio, \& Shapiro (1996) that some mixing could occur as a merger remnant recontracts to the main sequence. Due to the fragility of $\mathrm{Li}$, if some mixing of surface material does occur during the coalescence, it will at least dilute, and possibly also destroy, any lithium remaining in the stars' thin convective surface zones up to that time. One might suppose that mass transfer in a detached system also destroys $\mathrm{Li}$, though one could also imagine gentle mass-transfer processes where the rate is slow enough that the original envelope is not subjected to additional mixing and where the transferred matter itself does not undergo additional Li destruction. Of course, mass transfer via Roche lobe overflow in a detached system, or wind accretion from a more distant companion, involves mass from an evolved star which may have already depleted its surface Li due to single-star evolutionary processes. Consequently, the mass transferred may be already devoid of $\mathrm{Li}$, as in the scenario quantified by Norris et al. (1997c).

We also note the possibility that the accretor in a masstransfer system, or the progenitors of a coalescence, was (were) devoid of $\mathrm{Li}$ prior to that event. $\mathrm{Li}$ is (normally) preserved in halo stars only over the temperature range from the turnoff $\left(T_{\text {eff }} \simeq 6300 \mathrm{~K}\right)$ to about $T_{\text {eff }} \simeq 5600 \mathrm{~K}$, corresponding to a mass range from 0.80 down to $0.70 M_{\odot}$. Therefore, it is likely that any mass accretor, and certain that any merger remnant, now seen in this mass range began life as one (or two) stars with initial mass(es) less than $0.70 M_{\odot}$ and had already destroyed Li normally, as lower mass stars are known to do, prior to mass exchange. In such a scenario, it is not necessary for any $\mathrm{Li}$ to have been destroyed as a result of the blue straggler formation process itself, though this could occur as well.

\section{DISCUSSION}

In view of the distributions of the ultra-Li-deficient stars in the $T_{\text {eff }}-[\mathrm{Fe} / \mathrm{H}]$ plane, with four at the turnoff and four 100-200 K cooler, we consider whether all represent the same phenomenon or whether two distinct processes have been in operation. It is not a trivial matter to answer this question because we do not know with certainty what mechanism(s) has affected any of the stars. However, we explore a number of possibilities in the discussion that follows. Ignoring again the obvious blue straggler BD $+25^{\circ} 1981$, of the 111 stars shown in Figure 3, eight are ultra-Li-deficient. If all ultra-Li-poor stars have the same origin, then we should begin by restating the frequency of such Li-weak objects as $\simeq 7 \%$ of plateau stars, rather than $\simeq 5 \%$ as estimated previously when the parameter space was incompletely sampled, with strong metallicity and temperature dependences in that fraction.

\subsection{Do Ultra-Li-deficient Stars and Field Blue Stragglers Share a Common Origin?}

Historically, blue stragglers and ultra-Li-deficient stars have been regarded as separate phenomena. However, we have been driven to consider whether there is any astrophysical basis for this separation. One must ask whether the process(es) that gives rise to blue stragglers is capable of producing only stars whose mass is greater than that of the main sequence turnoff of a $\sim 13 \mathrm{Gyr}$ old population. If, as we think is reasonable, the answer is "no," then one may ask what the subturnoff-mass products of this process(es) would be. Our proposal is that they would be Li-deficient but otherwise difficult to distinguish from the general population. ${ }^{3}$

For ultra-Li-poor stars redder than the main-sequence turnoff, Hipparcos parallaxes have established that G186-26 is on the main sequence rather than on the subgiant branch. Of those at the turnoff, Wolf 550, G202-65, and $\mathrm{BD}+51^{\circ} 1817$ also have Hipparcos parallaxes; two are almost certainly dwarfs, while G202-65 is subject to larger uncertainties and may be more evolved (see Ryan et al. 2001, Table 2). The argument that the evolutionary rate of subgiants is too rapid to explain the high frequency of observed Li-deficient objects, which persuaded Norris et al. (1997c) to reject the proposition that they might be the redward-evolving (postturnoff) progeny of blue stragglers, is therefore redundant. However, the detection of several Li-weak stars at the bluest edge of the color distribution has prompted us to reexamine their possible association with blue stragglers.

We would describe G202-65 as "at" the turnoff rather than classify it as a blue straggler in the conventional sense, as it is only marginally hotter (bluer) than the mainsequence turnoff for its metallicity (see Fig. 3). Hobbs \& Mathieu (1991), on the other hand, classified it as a blue straggler, based presumably on the photometry of Laird, Carney, \& Latham (1988), which they referenced. (Indeed, Carney et al. 1994 declare it as a "blue straggler candidate," and Carney et al. 2000 treat it as one, though acknowledging at the same time that some normal stars may be included in this classification.) Our purpose is not to debate how this star should be classified but, rather, to underline the main suggestion of our work, that the blue straggler and halo ultra-Li-deficient stars may have a common origin. Although blue stragglers have historically been recognized because they are bluer than the main-sequence turnoff, it is essential to remember that stars that have accreted mass from a companion or that result from a coalescence can have a mass less than the current turnoff. Such stars would be expected to share many of the properties of blue stragglers but would not yet appear bluer than the turnoff. However, at some future time, once the main-sequence turnoff reaches lower masses, these nonstandard objects would lag the evolution of normal stars and hence appear bluer, showing canonical straggling behavior. Therefore, such stars might, for the present, be regarded as "blue-

\footnotetext{
${ }^{3}$ The likelihood of subturnoff-mass objects being produced by the bluestraggler-forming process is independently addressed in the model by Preston \& Sneden $(2000, \S 5.3)$, which came to our attention during finalization of this manuscript.
} 
stragglers-to-be," ${ }^{4}$ and our speculation is that the ultra-Lideficient halo stars in are in fact members of such a population. Note that this proposition is distinct from that of redward-evolving systems considered and rejected by Norris et al. (1997c).

If ultra-Li-deficient stars and blue stragglers are manifestations of the same process, then Li deficiency may be the only way of distinguishing subturnoff-mass blue-stragglersto-be from normal main-sequence stars, prior to their becoming classical blue stragglers. Mass transfer during their formation may also help clarify some of the unusual element abundances found by Norris et al. (1997c; see also Ryan et al. 1998). Whereas an appeal to extra mixing (in a single-star framework) to explain the Li depletion would not necessarily affect other elements, mass transfer in a binary with an asymptotic giant branch (AGB) donor may be capable of altering $s$-process abundances as well. In this regard, we recall that two of the ultra-Li-deficient stars studied by Norris et al. (1997c; also Ryan et al. 1998) had nonstandard $\mathrm{Sr}$ and $\mathrm{Ba}$ abundances. Mass transfer from a red giant branch (RGB) donor would presumably leave a different chemical signature. ${ }^{5}$

Some constraints on the progenitors of the Li-deficient stars may be obtained from their rotation rates and radial velocity variations. Webbink's (1976) calculations of a coalesced star $\left(M_{\text {total }}=1.85 M_{\odot}\right)$ show that a high rotation rate is maintained at least until it reaches the giant branch. In contrast, previously known blue stragglers appear not to have uncommonly high rotation rates (e.g., Carney \& Peterson 1981; Pritchet \& Glaspey 1991). This tends to argue against the blue stragglers as having originated from coalesced main-sequence contact binaries and points toward one of the other binary mass-transfer scenarios, unless mass loss (e.g., via Webbink's excretion disk) and magnetic breaking can dissipate envelope angular momentum during the main-sequence lifetime of a coalesced star. To spin down, stars must have a way of losing surface angular momentum. In single stars, most of this is believed to occur during the pre- and early main-sequence phase when magnetic coupling of the stellar surface to surrounding dust creates a decelerating torque on the star. It is not clear that two mature stars which merge will still have this coupling, because of the much lower mass-loss rates beyond the early stages of evolution (unless they produce an excretion disk) and lower magnetic field strengths. (See also discussion by Sills et al. 1997, § 5.5.) Leonard \& Livio (1995) have proposed that the merger product acquires the distended form of a pre-main-sequence-like star which then spins down as it again approaches the main sequence, losing angular momentum in much the same way as conventional pre-main-sequence stars. $^{6}$

\footnotetext{
${ }^{4}$ Independently, Carney et al. (2000) have noted this possibility, and models by Portegies Zwart (2000) predict the existence of such objects.

${ }^{5}$ Amongst very metal-poor stars with $[\mathrm{Fe} / \mathrm{H}]<-2.5$, as many as $25 \%$ have C overabundances (e.g., Norris, Ryan, \& Beers 1997a). At least some but not all of these (Norris, Ryan, \& Beers 1997b) have s-process anomalies. Detailed studies have yet to be completed, so it is unclear what fraction of stars are formed from anomalous material and what fraction became modified later in their life. Whilst we cannot presently rule out the possibility that the s-process anomalies seen in some ultra-Li-deficient stars were inherited at birth, our expectation is that mass transfer from a companion star will be a more common mechanism.

${ }^{6}$ Although stellar collisions will be rare for stars in the field, we should recall that most stars are probably born in clusters, and prior to cluster dissolution, collisions would have greater probability.
}

For the four stars observed in this work, three had previous radial velocity measurements accurate to $\simeq 1 \mathrm{~km} \mathrm{~s}^{-1}$ (Carney et al. 1994). The new measurements (Ryan et al. 2001, Table 2) showed residuals of $+1.0\left(\mathrm{BD}+51^{\circ} 1817\right)$, -3.3 (G202-65), and $-6.9 \mathrm{~km} \mathrm{~s}^{-1}$ (Wolf 550); compared with the expected radial velocity accuracy of $\sigma_{v}=0.3-0.7$ $\mathrm{km} \mathrm{s}^{-1}$, these are consistent with significant motion. Carney et al. (2000) indicate periods of 168-694 days for these systems, and low eccentricities, except for Wolf 550 $(e=0.3)$. Similarly, the metal-poor field blue straggler CS 22966- 043 has an orbital period of 319 days (Preston \& Landolt 1999). If the brighter component has a mass of 0.8 $M_{\odot}$ and its companion has a mass between 0.4 and $1.4 M_{\odot}$ (appropriate to a white dwarf), then the current semimajor axis of the system will be in the range $a=200-260 R_{\odot}$ (from Kepler's third law). ${ }^{7}$ Their second system, CS 29499 057, may have an even longer period of 2750 days, implying $a=900-1100 R_{\odot}$. The periods of these and Carney et al.'s systems, and hence their large current separations, are more compatible with mass loss from an evolved companion rather than being short-period systems in contact on the main sequence.

The evidence presented to date has argued against internal mixing alone as an adequate explanation for the ultraLi-deficient stars whose neutron-capture elements show abundance anomalies. Note, though, that certainly not all ultra-Li-deficient stars and blue stragglers exhibit neutroncapture element anomalies (Carney \& Peterson 1981; Norris et al. 1997c; Ryan et al. 1998). If mass transfer has occurred, systems in which $s$-process elements are abnormal would presumably indicate material originating with an AGB companion, whereas s-process-normal remnants would indicate mass transfer during an earlier stage of evolution (RGB) or from a pre-thermal-pulsing AGB mass donor. (We have no data on the $\mathrm{N}$ abundance, and the $\mathrm{CH}$ band in these stars is too weak to hope to measure the ${ }^{12} \mathrm{C} /{ }^{13} \mathrm{C}$ ratio.) Likewise, the rotation rates of both blue stragglers and ultra-Li-deficient stars are apparently normal, arguing against coalescences having already occurred on the main sequence. Of the three mechanisms found to be viable by Pritchet \& Glaspey (1991) and Hobbs \& Mathieu (1991), this leaves mass transfer from a companion as the only one remaining, if we are correct in speculating that the ultra-Li-deficient and blue straggler phenomena are manifestations of the same process.

\subsection{The Hot Stars in Isolation}

In the absence of an adequate theory for why eight otherwise normal halo stars (excluding the traditional blue straggler BD $+21^{\circ} 1981$ ) should have low (zero?) Li abundances, it may be useful to consider the hot subsample $(6200 \mathrm{~K} \lesssim$ $T_{\text {eff }} \lesssim 6300 \mathrm{~K}$ ) as a distinct group. Several possibilities then arise that might account for the observed $\mathrm{Li}$ deficiency, including diffusion (the sinking of $\mathrm{Li}$ to below the photosphere), the F-star Li dip, and an unknown process that may be responsible for depletion in some (but not all) disk stars. We consider each of these in turn. We note that the three Li-deficient stars with $T_{\text {eff }} \simeq 6300 \mathrm{~K}$ are confirmed binaries, whereas most cooler ones show no evidence of binary motion. The binary/single distinction between

\footnotetext{
${ }^{7}$ Carney et al. (2000) argue that all of their blue straggler observations are consistent with $0.55 M_{\odot}$ companions having a canonical white dwarf mass.
} 
warmer/cooler Li-depleted stars is pronounced; see Table 1, where the binary status (Carney et al. 1994, 2000; D. A. Latham 2000, private communication) is given in the final column. If such a dichotomy is maintained as more Li-poor systems are discovered, it may indicate a genuine difference in the origin of the turnoff and subturnoff systems.

\subsubsection{Diffusion}

Deliyannis, Demarque, \& Kawaler (1990) and Proffitt \& Michaud (1991) have computed the predicted effects of diffusion on the surface $\mathrm{Li}$ abundances of warm halo stars. Diffusion is more significant in hotter stars because their surface convective zone is thinner. The degree of depletion expected at $T_{\text {eff }} \sim 6300 \mathrm{~K}$ is a function of effective temperature, changing by $\simeq 0.2$ dex per $100 \mathrm{~K}$ in the former (for $\alpha=1.1)$ and $\simeq 0.2$ and greater than 0.2 dex per $100 \mathrm{~K}$ in the latter (for $\alpha=1.7$ and 1.5, respectively). This does not match the behavior observed (see Fig. 2). For comparison, our ultra-Li-poor stars are depleted by $\gtrsim 0.8$ dex. This alone appears to rule out diffusion as the explanation, except possibly for the lower $\alpha$ model of Proffitt \& Michaud. However, $\mathrm{Li}$ diffusion appears to have been inhibited in all other metal-poor samples (e.g., Ryan et al. 1996), so it would be unusual to see it suddenly present and with such effect only in isolated stars in our new sample.

\subsubsection{The F-Star Li Dip}

Boesgaard \& Tripicco (1986) and Hobbs \& Pilachowski (1988) showed that Li is severely depleted in Population I open cluster stars over the interval $6400 \mathrm{~K}<T_{\text {eff }}<7000 \mathrm{~K}$. Various explanations have been proposed, including mass loss (e.g., Schramm, Steigman, \& Dearborn 1990), diffusion (e.g., Turcotte, Richer, \& Michaud 1998), and slow mixing of various forms (e.g., Deliyannis \& Pinsonneault 1997), but none has been convincingly established as responsible, and several mechanisms may be acting in concert (e.g., Turcotte et al.). Whatever the correct explanation(s), is it possible that the hottest ultra-Li-deficient stars are encroaching on this regime and are affected by this phenomenon? Although this cannot be ruled out completely for the hot subset, especially since we have questioned the reliability of the $E(B-V)$ (and hence $T_{\text {eff }}$ ) values of the hottest Li-preserving stars in Figure 2, the onset of destruction in the F-star dip seems too gradual with $T_{\text {eff }}$ to explain the new data. The Hyades observations (Boesgaard \& Tripicco 1986) show a decrease of only 0.3 dex from 6200 to $6400 \mathrm{~K}$, substantially less than the $\gtrsim 0.8$ dex deficit in the ultra-metal-poor objects around $6300 \mathrm{~K}^{8}$ As noted above, Hipparcos parallaxes are available for five of the eight known ultra-Lideficient stars and, with the possible exception of G202-65, rule out the possibility that these stars are redward-evolving descendants of the Li dip.

\subsection{Anomalously Li-depleted Disk Stars}

Lambert, Heath, \& Edvardsson (1991) found that, in almost all cases, the low Li abundances in their Population I sample could be ascribed to their being evolved descendants of Li-dip stars or else being dwarfs exhibiting the $\mathrm{Li}$ depletion that increases toward lower temperature, as is normally associated with pre-main-sequence and/or main-

\footnotetext{
${ }^{8}$ The critic could object that there are deficiencies in comparing metalrich and metal-poor objects in this fashion. We would agree, but would also note that such a comparison is justifiable if only to show that the two behaviors are dissimilar.
}

sequence burning. Anomalously high Li depletions were found in only 1-3 cases out of some 26 old-disk stars and for a similar fraction of young-disk stars. Based on this fraction, Lambert et al. proposed that a new class of highly Li-depleted stars, comprising less than about $10 \%$ of the population, might exist. It is interesting to note that this proposal predated the discovery of ultra-Li-deficient halo dwarfs.

The uncertain number of cases stated above arises because Lambert et al. recognized that uncertainties in the stellar luminosities, and hence mass, could drive stars into or out of the region of importance. We now have the benefit of accurate Hipparcos parallaxes. These indicate that two of the seven stars highlighted by their study, HD 219476 and HR 4285, are indeed considerably more massive than reported in Lambert et al.'s tables and hence are probably descendants of the Li gap, thus reducing the number of genuine cases to two out of 26 old-disk stars, and 3 out of a similar number of young-disk stars. That is, the fraction of anomalously Li-depleted stars appears to be around $8 \%-10 \%$, albeit sensitive to small-number statistics. ${ }^{9}$ Ultra-Li-depleted Population I stars are also seen in young open clusters. They can be recognized, for example, in Figure 1 of Ryan \& Deliyannis (1995), where $\simeq 6 \%$ of the Hyades stars cooler than the F-star dip appear to be ultraLi-deficient.

Is it possible that the Li-depleted halo stars are of the same type? The lack of examples in the two Population I and Population II classes to compare with precludes a detailed analysis, but we note that we see Li deficiency in about $7 \%$ of halo objects, which is comparable to the ratio for the Population I objects. That is, the Population I and Population II examples could arise due to the same process, even though it remains unclear what that process is. We note, for completeness, that Ryan et al. (2001) showed that the kinematics of the new ultra-Li-depleted stars are clearly those of halo objects, and thus they genuinely belong to the halo population, despite their metallicities being close to those of the most metal-poor thick-disk stars.

The stars remaining on Lambert et al's list of unusually Li-deficient objects are HR 3648, HR 4657, HR 5968, HR 6541, and HD 30649. Upon searching the literature for evidence of binarity or abundance anomalies in these systems, we found that not only was HR 4657 an 850 day period binary, but Fuhrmann \& Bernkopf (1999) had also been driven to consider this star as a blue straggler. It has an unexpectedly high rotational velocity (in contrast to the blue stragglers studied by Carney \& Peterson 1981). There is no evidence of $s$-process anomalies, but other unusual characteristics of the system include an observable soft $\mathrm{X}$-ray flux and the very likely association of this object with GRB 930131. HR 3648 (= $16 \mathrm{UMa}=$ HD 79028) is a 16.2 day period chromospherically active single-lined spectroscopic binary (Basri, Laurent, \& Walter 1985). HD 30649 (= G81-38) and HR 6541 (=HD 159332), in contrast, show no significant evidence of binarity (Carney et al. 1994). HR $5968(=\rho \mathrm{CrB})$ does not appear to have a stellar companion, though it does have a planetary companion (Noyes et al. 1997), but Ryan (2000) argues that $\mathrm{Li}$ in this star is not anomalous. HR 3648 and HR 4657 have Ba abundance measurements from the study by Chen et al. (2000). The

\footnotetext{
${ }^{9}$ Errors in temperature could reduce these cases further.
} 
latter also has been observed by Fuhrmann \& Bernkopf (1999), but neither star appears abnormal in this element.

\section{IMPLICATIONS AND SUMMARY}

Ryan et al. (1999) have argued that the ultra-Li-deficient halo stars are distinct from the majority of halo stars that occupy the Spite plateau, and, in particular, that they do not merely represent the most extreme examples of a continuum of $\mathrm{Li}$ depletion. If the association with blue stragglers (or, for that matter, any distinct evolutionary phenomenon) is correct, then the mechanism for their unusual abundances will at last be understood, and they will be able to be neglected with certainty from future discussion of the Spite plateau.

In the present work, we have proposed and discussed the possibility that ultra-Li-depleted halo stars and blue stragglers are manifestations of the same phenomenon, and described the former as "blue-stragglers-to-be." We proposed that their $\mathrm{Li}$ was destroyed either during the formation process of blue stragglers or during the normal single-star evolutionary processes of their precursors, namely, during pre-main-sequence and/or main-sequence phases of low-mass stars or during post-main-sequence evolution of mass donors, as in the scenario quantified by Norris et al. (1997c). We note that in a study carried out separately but over the same time period as ours, Carney et al. (2000) have examined the orbital characteristics of blue stragglers and have been driven toward similar considerations. There are clearly still details to be clarified, but our two groups appear to be converging on a view unifying blue stragglers and ultra-Li-deficient systems.

Because there are numerous observational and theoretical issues surrounding this unified view, we seek to clarify the main arguments and possibilities using an itemized summary.

\section{Observations}

a) In a study of 18 halo stars with $-2 \lesssim[\mathrm{Fe} / \mathrm{H}] \lesssim-1$ and $6000 \mathrm{~K} \lesssim T_{\text {eff }} \lesssim 6400 \mathrm{~K}$, we have found four ultra-Lideficient objects, i.e., a $22 \%$ detection rate.

$b$ ) The fraction of ultra-Li-deficient stars is very much higher amongst the hottest and most metal-rich halo mainsequence stars $(\simeq 20 \%)$ than amongst cooler and more metal-poor ones $(\simeq 5 \%)$.

c) Ultra-Li-deficient stars exist both at the turnoff and cooler than the turnoff and with well-determined mainsequence luminosities from Hipparcos.

d) All of the turnoff ultra-Li-deficient halo stars, but none of the subturnoff ultra-Li-deficient halo stars, appear to be binaries. This may indicate that two different mechanisms are causing the halo ultra-Li-deficient phenomenon.

\section{Theoretical Framework}

a) Blue stragglers may form from several mechanisms but seem to require at least one of either complete mixing,

\footnotetext{
${ }^{10}$ Coalescence may be between the components of an existing binary, possibly having been hardened via interactions with a third star, or through direct collisions (which may also be moderated by binary interactions).
}

binary mass transfer, or coalescence ${ }^{10}$ (Hobbs \& Mathieu 1991; Pritchet \& Glaspey 1991).

\section{Origins}

a) We speculate that ultra-Li-deficient stars and blue stragglers are manifestations of the same process, and that subturnoff-mass ultra-Li-deficient stars may be regarded as "blue-stragglers-to-be."

b) Li could be destroyed at several stages: (i) in a masstransfer event which induces extensive mixing; (ii) by singlestar evolutionary processes (convective mixing) in a post-main-sequence mass donor; (iii) by single-star evolutionary processes (mixing) in pre-main-sequence (or possibly main-sequence) low-mass stars prior to their gaining mass.

c) Mass-transfer scenarios from an AGB star seem better able to explain the unusual neutron-capture element ratios sometimes seen in ultra-Li-depleted stars (Norris et al. $1997 \mathrm{c})$ than internal mixing, since $\simeq 0.8 M_{\odot}$ corehydrogen-burning stars are not expected to process neutron-capture elements. This argues against internal mixing as the sole explanation for the existence of ultra- $\mathrm{Li}$ depleted stars with unusual neutron-capture abundances. (Mass transfer from pre-AGB [most likely RGB] donors would produce the stars with normal neutron-capture abundances.)

d) Coalesced binaries are expected to maintain high rotation rates until they reach the giant branch, but neither blue stragglers nor ultra-Li-depleted halo stars have high rotation rates. This argues against coalescence of a binary as the explanation for these objects unless they have spun down.

$e$ ) The orbital periods of metal-poor field blue stragglers (Preston \& Landolt 1999; Carney et al. 2000) suggest current semimajor axes in the range $200-1100 R_{\odot}$, arguing against these being coalescing stars (unless they began their lives as triple systems).

f) The arguments against solely internal mixing, and against coalescence of main-sequence contact binaries, leaves mass transfer as the most viable mechanism for field binaries. This is not to say that $\mathrm{Li}$ was destroyed during the transfer; it may have been destroyed by single-star mechanisms already.

g) The observed $d A(\mathrm{Li}) / d T_{\text {eff }}$ is too steep compared with models of diffusion to be due to that process.

h) The observed $d A(\mathrm{Li}) / d T_{\text {eff }}$ is too steep compared with the Hyades data to be due to the F-star Li dip.

i) The halo ultra-Li-deficient stars could be related to the Population I anomalously Li-depleted stars identified in the field by Lambert et al. (1991) and also seen in open clusters.

j) Hipparcos parallaxes rule out the possibility that the ultra-Li-deficient stars are redward-evolving postturnoff stars. They have not descended from the F-star Li dip.

\section{Implications}

a) Severe Li depletion may be the (only?) signature of subturnoff-mass blue stragglers. The halo population fraction comprising ultra-Li-poor stars is $7 \%$.

b) Understanding the ultra-Li-depleted stars as resulting from a distinct process (not normally affecting single stars) would eliminate the need to include them in discussions of processes affecting the evolution of normal Spite plateau stars and would explain why they appear so radically different from the vast majority of halo stars (Ryan et al. 1999). 
The authors gratefully acknowledge the support for this project given by the Australian Time Assignment Committee (ATAC) and Panel for the Allocation of Telescope Time (PATT) of the AAT and WHT, respectively, and for practical support given by the staff of these facilities. They also express gratitude to D. A. Latham and B. W. Carney for conveying the results of their program in advance of publication and to an anonymous referee for his/her comments that helped us clarify our arguments. S. G. R. sends a special thanks to colleagues at the University of Victoria: to C. J. Pritchet for a most memorable snow-shoeing expedition on 1991 February 10 during which Li deficiency in blue stragglers was discussed, to D. A. VandenBerg for discussing and supplying isochrones, and to F. D. A. Hartwick for once asking whether there were blue stragglers in the halo field. T. C. B. acknowledges partial support from grant AST 95-29454 from the National Science Foundation.
Basri, G., Laurent, R., \& Walter, F. M. 1985, ApJ, 298, 761

Bergbusch, P. A., \& VandenBerg, D. A. 1992, ApJS, 81, 163

Boesgaard, A. M., \& Tripicco, M. J. 1986, ApJ, 302, L49

Bonifacio, P., \& Molaro, P. 1997, MNRAS, 285, 847

Carney, B. W., Latham, D. W., Laird, J. B., \& Aguilar, L. A. 1994, AJ, 107, 2240

Carney, B. W., Latham, D. W., Laird, J. B., Grant, C. E., \& Morse, J. A. 2000, preprint

Carney, B. W., \& Peterson, R. C. 1981, ApJ, 251, 190

Chaboyer, B. 2000, The Galactic Halo: From Globular Clusters to Field Stars, ed. A. Noels, P. Magain, D. Caro, E. Jehin, G. Parmentier, \& A. Thoul (Liège: Inst. d'Astrophysique et de Géophysique)

Chaboyer, B., Demarque, P., \& Pinsonneault, M. H. 1995, ApJ, 441, 876

Chen, Y. Q., Nissen, P. E., Zhao, G., Zhang, H. W., \& Benoni, T. 2000, A\&AS, 141, 491

Deliyannis, C. P. 1995, The Light Element Abundances, ed. P. Crane (Berlin: Springer), 395

Deliyannis, C. P., Demarque, P., \& Kawaler, S. D. 1990, ApJS, 73, 21

Deliyannis, C. P., King, J. R., Boesgaard, A. M., \& Ryan, S. G. 1994, ApJ, 434, L71

Deliyannis, C. P., \& Pinsonneault, M. H. 1997, ApJ, 488, 836

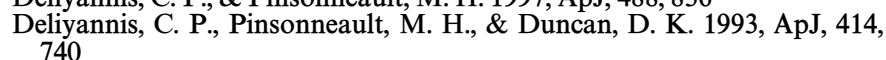

Ferraro, F. R., Fusi Pecci, F., \& Bellazini, M. 1995, A\&A, 294, 80

Ferraro, F. R., Palterinieri, B., Rood, R. T., \& Dorman, B. 1999, ApJ, 522, 983

Fuhrmann, K., \& Bernkopf, J. 1999, A\&A, 347, 897

Green, E. M., Demarque, P., \& King, C. R. 1987, The Revised Yale Isochrones and Luminosity Functions (New Haven: Yale Univ. Press)

Hobbs, L. M., \& Mathieu, R. D. 1991, PASP, 103, 431

Hobbs, L. M., \& Pilachowski, C. 1988, ApJ, 334, 734

Hobbs, L. M., Welty, D. E., \& Thorburn, J. A. 1991, ApJ, 373, L47

Jeffries, R. D. 1999, MNRAAS, 309, 189

Jones, B. F., Fischer, D., \& Soderblom, D. R. 1999, AJ, 117, 330

Laird, J. B., Carney, B. W., \& Latham, D. W. 1988, AJ, 95, 1843

Lambert, D. L., Heath, J. E., and Edvardsson, B. 1991, MNRAS, 253, 610

Leonard, P. J. T. 1989, AJ, 98, 217

Leonard, P. J. T., \& Livio, M. 1995, ApJ, 447, L121

Lombardi, J. C., Jr., Rasio, F. A., \& Shapiro, S. L. 1996, ApJ, 468, 797

Martín, E. L., \& Claret, A. 1996, A\&A, 306, 408

Mateo, M., Harris, H. C., Nemec, J., \& Olszewski, E. W. 1990, AJ, 100, 469

Montalbán, J., \& Schatzman, E. 1996, A\&A, 305, 513

Norris, J. E., Beers, T. C., \& Ryan, S. G. 2000, ApJ, 540, 456

Norris, J. E., Ryan, S. G., \& Beers, T. C. 1997a, ApJ, 488, 350

. 1997b, ApJ, 489, L169

\section{REFERENCES}

Norris, J. E., Ryan, S. G., Beers, T. C., \& Deliyannis, C. P. 1997c, ApJ, 485, 370

Noyes, R. W., Jha, S., Korzennik, S. G., Krockenberger, M., Nisenson, P., Brown, T. M., Kennelly, E. J., \& Horner, S. D. 1997, ApJ, 483, L111

Piotto, G. 2000, The Galactic Halo: From Globular Clusters to Field Stars, ed. A. Noels, P. Magain, D. Caro, E. Jehin, G. Parmentier, \& A. Thoul (Liège: Inst. d'Astrophysique et de Géophysique)

Portegies Zwart, S. 2000, Proc. Conf. on the Influence of Binaries on Stellar Population Studies, ed. D. Vanbeveren (Dordrecht: kluwer), in press

Preston, G. W., \& Landolt, A. U. 1999, AJ, 118, 3006

Preston, G. P., \& Sneden, C. 2000, AJ, 120, 1014

Pritchet, C. J., \& Glaspey, J. W. 1991, ApJ, 373, 105

Proffitt, C. R., \& Michaud, G. 1991, ApJ, 371, 584

Rebolo, R., Molaro, P., \& Beckman, J. E. 1988, A\&A, 192, 192

Ryan, S. G. 2000, MNRAS, 316, L35

Ryan, S. G., Beers, T. C., Deliyannis, C. P., \& Thorburn, J. A. 1996, ApJ, 458,543

Ryan, S. G., \& Deliyannis, C. P. 1995, ApJ, 453, 819

Ryan, S. G., Kajino, T., Beers, T. C., Suzuki, T., Romano, D., Matteucci, F., \& Rosolankova, K. 2001, ApJ, in press

Ryan, S. G., Norris, J. E., \& Beers, T. C. 1998, ApJ, 506, 892 .1999, ApJ, 523, 654

Sandage, A. R. 1953, AJ, 58, 61

Sandquist, E. L., Bolte, M., \& Hernquist, L. 1997, ApJ, 477, 335

Schatzman, E. 1993, A\&A, 279, 431

Schramm, D. N., Steigman, G., \& Dearborn, D. S. P. 1990, ApJ, 359, L55

Sills, A. P., Bailyn, C. D., \& Demarque, P. 1995, ApJ, 455, L163

Sills, A., Lombardi, J. C., Jr., Bailyn, C. D., Demarque, P., Rasio, F. A., \& Shapiro, S. L. 1997, ApJ, 487, 290

Spite, F., \& Spite, M. 1982, A\&A, 115, 357

Spite, M., Maillard, J. P., \& Spite, F. 1984, A\&A, 141, 56

Spite, M., Molaro, P., François, P., \& Spite, F. 1993, A\&A, 271, L1

Spite, M., Spite, F., Cayrel, R., Hill, V., Depagne, E., Nordstrom, B., Beers, T. \& Nissen, P. E. 2000, in IAU Collog. 198, The Evolution of the Light Elements, ed. L. da Silva, R. de Medeiros, \& M. Spite (ASP Conf Ser.; San Francisco: ASP), in press

Stryker, L. L. 1993, PASP, 105, 1081

Thorburn, J. A. 1992, ApJ, 399, L83 . 1994, ApJ, 421, 318

Thorburn, J. A., \& Beers, T. C. 1993, ApJ, 404, L13

Turcotte, S., Richer, J., \& Michaud, G. 1998, ApJ, 504, 559

Ventura, P., Zeppieri, A., Mazzitelli, I., \& D’Antona, F. 1998, A\&A, 331, 1011

Webbink, R. F. 1976, ApJ, 209, 829 